\title{
Modeling dissimilar components of the eclipsing binary EK Cep:
}

\section{Does the primary star have a rapidly rotating core?}

\begin{abstract}
M. Yildiz
Ege University, Department of Astronomy and Space Sciences, Bornova, 35100 İzmir, Turkey

Received 20 January 2003/ Accepted 18 July 2003

Abstract. In this study we propose to explain the discrepancy between classical models and the observational data of early type stars in eclipsing binaries by the existence of a rapidly rotating core in the primary, rather than by invoking a low metal abundance. Our claim is based on the analysis of the double lined eclipsing binary EK Cep, on which a strong constraint is put by its apsidal motion. We constructed models both with and without rotation for the components of the system. If the stars do not rotate, then, considering (i) that both components have the same chemical composition and age, and (ii) that the primary star is exactly at the zero-age main-sequence point, we derive $X=0.614$ and $Z=0.04$ for respectively the hydrogen and heavy element abundances, with a mixing-length parameter $\alpha=1.30$, somewhat lower than that calibrated on the Sun. These values satisfy all the observational constraints at the single age of $26 \mathrm{Myr}$, except the luminosity and radius of the primary, and they are also in good agreement with the observed chemical evolution in the solar neighborhood. Since the observed luminosity and radius of EK Cep A are less than those predicted by a non-rotating model, we deduce that this star must have a rapidly rotating core, while its envelope is synchronized with the orbital motion due to tidal interaction. To achieve perfect agreement between the rotating model of this star and the observations, the requirement is that the central region rotates about 65 times faster than the synchronized envelope, which contains $48 \%$ of the star's total mass. We describe the effect of such differential rotation on the location of the star in the HR diagram, and compare it with that of rotation caused by contraction alone. We confirm also that such rapid rotation may account for the spread which is observed in the isochrones of open clusters.
\end{abstract}

Key words. stars: interiors - stars: rotation - stars: individual: EK Cep - stars: evolution - stars: abundances binaries: eclipsing

\section{Introduction}

Current models for stars in the well known double lined eclipsing binaries (Claret et al. 1995; Claret 1997; Pols et al. 1997; Andersen et al. 1993) indicate that systems with early type components have low heavy element $(Z)$ and high hydrogen $(X)$ abundances, in contrast to systems with late type components. This result is against our understanding of the chemical evolution of the galactic disk, since systems with early type stars on average are younger than systems with late type stars, and, therefore they should have, on average, higher $Z$ values.

To overcome this contradiction, we may ask whether these stars are correctly modeled by the standard theory. Confident that current evolution codes, such as ours (Y1ldiz 2000), yield satisfactory results for the solar interior, it is natural to question the modeling of early type stars. If so, what is the problem with early type stars? More specifically, which of their properties lead to the prediction of low $Z$ and high $X$ ? The answer is that early type stars in some young eclipsing binaries, such as EK Cep, DI Her, GG Lup, GG Ori, have radii and luminosities

\footnotetext{
^ e-mail: yildiz@astronomy.sci.ege.edu.tr
}

which are too small, smaller even than their zero-age mainsequence (ZAMS) models, when assuming a reasonable chemical composition (e.g., the solar composition). Leaving aside the adoption of low $Z$ and high $X$ values, this problem can be solved by assuming that these stars possess a rapidly rotating core.

The present study concentrates on the eclipsing binary EK Cep, whose dissimilar components are very well known observationally (Andersen 1991, hereafter A91). The system displays apsidal motion and its secondary is a pre-main sequence (PMS) star. From the analysis based on the photoelectric light curve first obtained by Ebbighausen (1966), and on the first spectrographic observation by Tomkin (1983), the fundamental physical parameters of the components were determined very precisely. A debate on the system was fueled by the large radius of the secondary, given its mass. Popper (1987) supported Tomkin's conclusion about this anomaly, namely that EK Cep B was a contracting PMS star, while EK Cep A was on the ZAMS, and he proposed to observe $\mathrm{Li} \mathrm{I}$ in the spectrum of the secondary for the ultimate confirmation of its evolutionary phase. Martín \& Rebolo (1993, hereafter MR93) 
carried out the spectroscopic observation in the Li I $6708 \AA$ region and found that the lithium abundance of the secondary is $\log N(\mathrm{Li})=3.1 \pm 0.3$, which is very close to $\mathrm{Li}$ abundance of the PMS stars. Therefore, the secondary star of EK Cep with a mass of $1.121 \pm 0.012 M_{\odot}$ and a radius of $1.315 \pm 0.006 R_{\odot}$ is doubtlessly in the PMS phase. However, in their recent study on eclipsing binary systems, Ribas et al. (1998) redetermined the effective temperatures of the component stars of EK Cep, but they pointed that the appearing inconsistent results for these two components arise from the PMS nature of the secondary. Therefore, we have adopted the values for EK Cep listed in A91 as fundamental properties for the component stars.

On the other hand, MR93 attempted to determine also iron abundance using several lines in the spectra of EK Cep's components. Unfortunately, even leaving aside how well the total heavy element abundance is represented by iron abundance only, these values spread too much to constrain total metal abundance of the system.

Claret et al. (1995) constructed models for the components of EK Cep, adopting the mixing-length parameter $\alpha=1.50$. They fitted the radius of the two components for an age of $20 \mathrm{Myr}$, and found a good agreement with the observed apsidal advance rate, for $Z=0.015$.

The method and outcomes of our study are totally different from Claret et al. By fitting the radius and the luminosity of EK Cep B, and assuming that the two components have the same age, we establish its chemical composition $(X$ and $Z)$ and its mixing-length parameter $\alpha$. Then, to reconcile this composition with the observed radius and luminosity of the primary, we allow it to have a rapidly rotating core, of which we determine the size and the angular velocity.

The stellar modeling and the fitting procedure are described in Sect. 2, and our alternative rotating model for EK Cep A is presented in Sect. 3. In Sect. 4, we discuss the results and make an attempt to relate chemical peculiarities of stars to their internal rotational properties. In this section also, we show quantitatively that the width of the MS in the HRD of open clusters, attributed to the overshooting process, is well explained by rotation alone. Finally, concluding remarks are given in Sect. 5.

\section{Models for the components of EK Cep}

We model the pre-main-sequence and main-sequence evolution of the two components of EK Cep, adopting various chemical compositions for both components, and different values of $\alpha$ for EK Cep B only. The characteristics of our code were already described by Yıldız (2000) and Yıldız \& Kızıloğlu (1997), and therefore we shall not enter into details. Our equation of state uses the approach of Mihalas et al. (1990) in the computation of the partition functions (and their derivatives with respect to density and temperature) of hydrogen and helium; it takes into account ionization and internal energy of the eight most abundant heavy elements as well as the so-called $\tau$-effect (Harris et al. 1960). The radiative opacity is derived from Iglesias et al. (1992), completed by the low temperature tables of Alexander $\&$ Ferguson (1994). For the nuclear reactions rates we use the analytic expressions given by Caughlan \& Fowler (1988), and

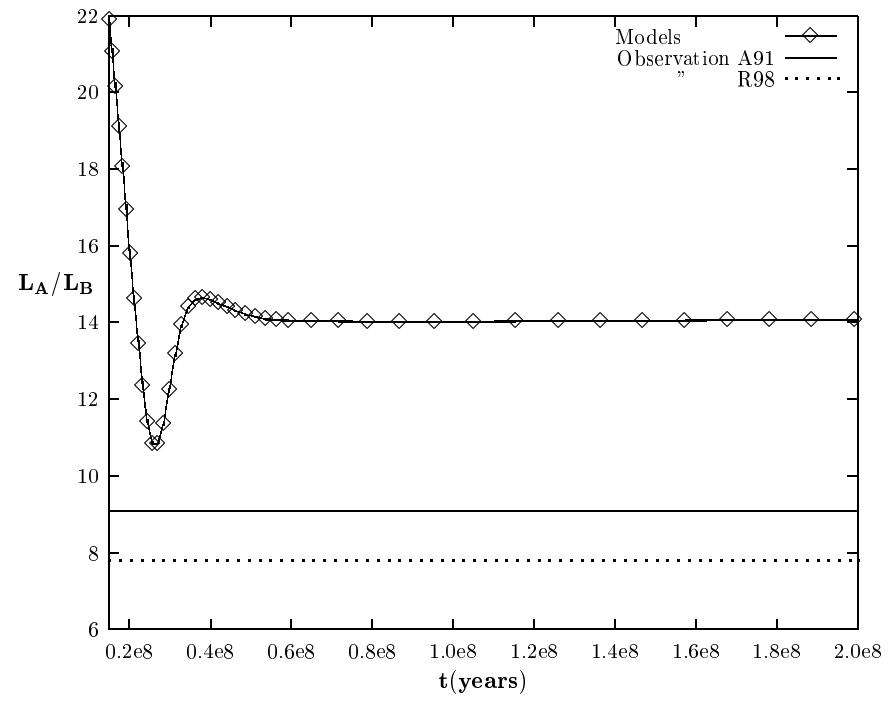

Fig. 1. Ratio between model luminosities of EK Cep A and EK Cep B as a function of time. The solar chemical composition and $\alpha$ are used for the models. The horizontal solid and dotted lines represent observed ratios by $\mathrm{A} 91$ and $\mathrm{R} 98$, respectively.

we employ the standard mixing length theory (Böhm-Vitense 1958).

Because the chemical composition has not yet been determined from direct observation, we assume solar composition. For the solar composition as well as for $\alpha$, we adopted values from Model 3 in Y1ldız (2000), in which diffusion of hydrogen and heavy elements are considered: $X=0.705, Z=0.02$ and $\alpha=1.88\left(\mathrm{SET}_{\odot}\right)$.

With these values, evolutionary models of the two components of EK Cep are constructed. None of the evolutionary tracks predicted by the models passes through the observed positions in the HRD (see Fig. 3): the observed position of the primary star is below its computed evolutionary track, the observed position of the secondary lies above its evolutionary track. However, in comparing theoretical and observational results, an important consideration is the ratio of luminosities and radii of the two components, rather than their individual values. For example, in Fig. 1, luminosity of model with $\mathrm{SET}_{\odot}$ for EK Cep A divided by that for EK Cep B $\left(l_{\mathrm{AB}} \equiv L_{\mathrm{A}} / L_{\mathrm{B}}\right)$ is plotted as a function of time, where horizontal lines represent observed ratios by A91 (solid line) and by R98 (dotted line). The differences between the theoretical and observed ratios here in Fig. 1 are so large that they can not be completely removed by some small modifications to the distance and/or to the chemical composition of the system. The minimum of the ratio (10.8) derived from the models corresponds to the point at which the luminosity of EK Cep B is maximum in PMS phase. Indeed, a similar situation is valid also for ratio of the radii $\left(r_{\mathrm{AB}} \equiv R_{\mathrm{A}} / R_{\mathrm{B}}\right)$ and we have a local minimum at that point (see Fig. 2). However, the difference between theoretical and observational $r_{\mathrm{AB}}$ can in principle be removed by decreasing calibration parameter $\alpha$ of the secondary star (see below).

In the following subsections, we will discuss the model properties of the component stars in more detail. To see how their properties change with $X$ and $Z$, we use three sets of chem- 


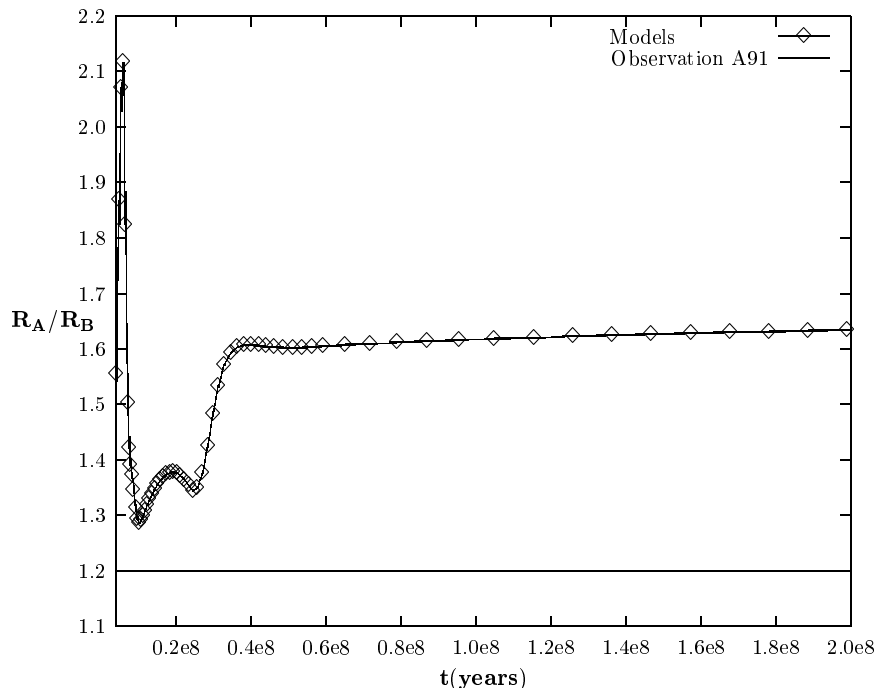

Fig. 2. The same as Fig. 1, but for the ratio between the radii.

ical composition: $\operatorname{SET}_{\odot}(X=0.705, Y=0.275, Z=0.02)$, SET1 (0.605, 0.355, 0.04), SET2 (0.785, 0.195, 0.02).

\subsection{Models for EK Cep A}

From the ZAMS models of the primary built with these three sets of chemical composition, we compute the numerical derivatives of luminosity and radius (in solar units) with respect to hydrogen and heavy element abundances:

$\frac{\Delta L}{\Delta X}=-67, \quad \frac{\Delta L}{\Delta Z}=-414$,

$\frac{\Delta R}{\Delta X}=0.31, \quad \frac{\Delta R}{\Delta Z}=12$.

We use them to fit the model with the observed radius and luminosity, and obtain $X=0.765, Z=0.0155$ (SET A).

Equation (2) demonstrates why models for early type stars, in general, require low $Z$ : near the ZAMS, the radius of a model for a given mass depends almost only on $Z$. If the observed radius of a star is less than the radius of its ZAMS model with the solar values, then, from a standard point of view, there is no other solution than decreasing $Z$. However, we should see whether this is valid also for EK Cep B.

\subsection{Models for EK Cep B}

In Fig. 3, models for the secondary star are plotted in the HRD; in all the models the mixing-length parameter is $\alpha=1.88$. The model with $\operatorname{SET}_{\odot}(Z=0.02$, solid line with $\diamond)$ and the model with SET1 $(Z=0.04$, dotted line with +$)$ have very similar evolutionary tracks in the HRD. These are blue-shifted with respect to the observational point, but we see that their maximum luminosity is close to the observed luminosity. On the other hand, the helium poor model (SET2, solid line with $\square$ ) has a much lower luminosity. From these three models, we find how

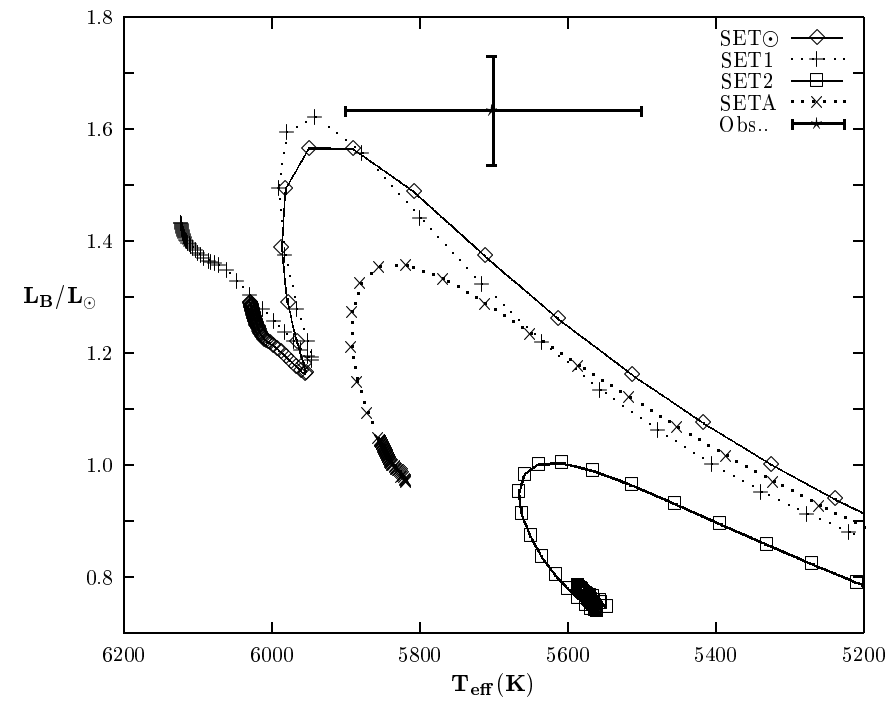

Fig. 3. Evolutionary paths of EK Cep B with various chemical compositions (the solar mixture for heavy elements), together with the observed positions in the HRD.

the maximum luminosity reached just before the MS and the corresponding radius depend on $X$ and $Z$ :

$\begin{array}{ll}\frac{\Delta L_{\mathrm{Max}}}{\Delta X}=-7.1, & \frac{\Delta L_{\mathrm{Max}}}{\Delta Z}=-33, \\ \frac{\Delta R_{\mathrm{Max}}}{\Delta X}=-1.6, & \frac{\Delta R_{\mathrm{Max}}}{\Delta Z}=-8.1 .\end{array}$

In Fig. 3, we also show the model with SET A, the composition we derived above for the primary (dotted line with $\times$ ): this model is so far from the observed position in the HRD that it can not be considered as a solution.

As suggested earlier, the difference between the theoretical and the observational $r_{\mathrm{AB}}$ (see Fig. 2) can be removed by adopting a smaller value (than the solar value 1.88) for $\alpha$. However, to do that, we need to know also numerical derivatives of the maximum luminosity and the radius with respect to $\alpha$. From the models of EK Cep B with different $\alpha$, we determine these derivatives:

$\frac{\Delta R_{\mathrm{Max}}}{\Delta \alpha}=-0.20, \quad \frac{\Delta L_{\mathrm{Max}}}{\Delta \alpha}=-0.08$.

Since the model radius is inversely proportional to $\alpha$, but its luminosity barely depends on it, for a given composition, the evolutionary track will be horizontally shifted toward the cool part of the HRD as $\alpha$ decreases. A decrease in $\alpha$ of 0.3 causes a reduction of $T_{\text {eff }}$ of about $100 \mathrm{~K}$, at least in the vicinity of the MS.

Since the secondary star has a rather large radius and high luminosity, we may assume that it is very close to the point where these parameters reach their maximum, just before the MS. Then, from the models of EK Cep B, we have two equations with three unknowns, namely, $\delta X, \delta Z$ and $\delta \alpha$ :

$L_{\mathrm{obs}}=L_{\mathrm{o}}+\frac{\Delta L_{\mathrm{Max}}}{\Delta X} \delta X+\frac{\Delta L_{\mathrm{Max}}}{\Delta Z} \delta Z+\frac{\Delta L_{\mathrm{Max}}}{\Delta \alpha} \delta \alpha$,

$R_{\mathrm{obs}}=R_{\mathrm{o}}+\frac{\Delta R_{\mathrm{Max}}}{\Delta X} \delta X+\frac{\Delta R_{\mathrm{Max}}}{\Delta Z} \delta Z+\frac{\Delta R_{\mathrm{Max}}}{\Delta \alpha} \delta \alpha$. 
The solution of Eqs. (6) and (7) require one more constraint. If we assume, for example, solar metallicity, then $\delta Z=0$ and we find a slightly higher hydrogen abundance and a significantly smaller convective parameter (SET B) than the solar values:

$X=0.71, \quad \alpha=1.27$.

Thus, at an age of 28.5 Myr, model of EKCep B with these values perfectly satisfies the observational constraints relevant to EKCep B. On the other hand, $l_{\mathrm{AB}}$ and $r_{\mathrm{AB}}$ computed from models with SET B are larger than the observed ratios, $11 \%$ and $3 \%$, respectively; to this we will return later.

\subsection{Estimation of the chemical composition and $\alpha$ based on coevality}

If the standard theory of stellar evolution were to work, we didn't then really need any additional constraint on this system, such as the solar metallicity. We could then very well write down two more similar equations in addition to Eq. (7), one for radius of the primary and the other for $l_{\mathrm{AB}}$. From the solution of these three equations, which is valid even when the distance to the system was unknown, we get very small $X$ and very large $\alpha$ :

$X=0.24, \quad Z=0.029, \quad \alpha=4.7$.

These values of $X$ and $\alpha$ are so extreme that the standard theory of stellar evolution is invalid in this case.

An additional constraint is needed. Since the observed value of $l_{\mathrm{AB}}$ is less then the minimum of its theoretical value, it is reasonable to suppose, as the additional constraint, that the primary is exactly at the ZAMS point where the luminosity is minimum. Thus, the third equation is obtained from the time difference $(\tau)$ between two different evolutionary phases of models for the component stars:

$\tau=t_{1}-t_{2}$

where $t_{1}$ is the ZAMS age of EK Cep A and $t_{2}$ refers to an age when the model of EK Cep B has its maximum luminosity before the MS. Since the system EK Cep has a single age, Eq. (10) should be equal to zero and thus

$0=\tau_{\mathrm{o}}+\frac{\Delta \tau}{\Delta X} \delta X+\frac{\Delta \tau}{\Delta Z} \delta Z+\frac{\Delta \tau}{\Delta \alpha} \delta \alpha$.

From the model properties of EK Cep A and B discussed above, numerical derivatives of $\tau$ (in units of $10^{7} \mathrm{yr}$ ) are computed as

$\frac{\Delta \tau}{\Delta X}=-4.7, \frac{\Delta \tau}{\Delta Z}=7.8, \frac{\Delta \tau}{\Delta \alpha}=0.21$

The solution of these three equations yield the following values (SET E):

$X=0.614, \quad Z=0.0395, \quad \alpha=1.30$.

The ratio of $\Delta Y$ to $\Delta Z$ is 3.64 and this is consistent with the observed chemical evolution of the solar neighborhood (Tosi 1995). The age of the system is found to be $26 \mathrm{Myr}$ when a rapidly growing convective core at the center of EK Cep B is about to form or has just been formed. However, the differences between the observed and the theoretical values of $l_{\mathrm{AB}}$ and $r_{\mathrm{AB}}$ are $19 \%$ and $13 \%$ of the observed ratios, respectively.

Indeed, the structure of a model changes slowly in time when it is near to the ZAMS. Therefore, we need to find out the chemical composition and $\alpha$ when the primary is close to, but not exactly at, the ZAMS point. For example, supposing that EK Cep A reached the ZAMS point $1 \mathrm{Myr}$ ago, then this solution (SET F) is found:

$X=0.630, \quad Z=0.036, \quad \alpha=1.31$.

Now, the minimum differences between the observed and the theoretical values of $l_{\mathrm{AB}}$ and $r_{\mathrm{AB}}$ are $16 \%$ and $12 \%$ of the observed ratios, respectively. Whether EK Cep B is near to or at the ZAMS point, these differences are too large to be solved by conventional means, such as overshooting and/or semi-convection.

\section{Rotating models for EK Cep A}

The above mentioned discrepancy can be eliminated by assuming a rapidly rotating core for EK Cep A. Indeed, given that the system is very young, differential rotation could arise due to the following two reasons: 1) the central regions contract more and consequently rotate faster than outer regions, provided that angular momentum transfer is slow enough; 2) according to Zahn's theory (1977) on tidal interaction in binary stars, gravity waves are forced in the radiative interior, which are dissipated in the outer envelope; since they transport negative angular momentum, they bring the outer layers to synchronism with the orbital motion (see also Goldreich \& Nicholson 1989).

Thus, we have good reasons to believe that the rapidly rotating core for EK Cep A is a plausible solution. The effect of rotation on the stellar structure is considered here in its simplest form: with the usual meanings of the symbols, the hydrostatic equation can be written as (Kippenhahn et al. 1970)

$\frac{\mathrm{d} P}{\mathrm{~d} r}=-\frac{G M_{r} \rho}{r^{2}}\left(1-\Lambda_{r}\right)$

where

$\Lambda_{r}=\frac{2}{3} \frac{\omega^{2} r^{3}}{G M_{r}}$

is the ratio between the averaged centrifugal force and the gravity. A rotating star evolves as if it were a non-rotating, less massive star, depending upon details of the angular momentum distribution. The central density (temperature) of a model with large mean $\omega$ is larger (lower) than that of a model with small $\omega$. Since energy generating nuclear reactions are more sensitive to temperature than to density, a rotating model is always less luminous than its non-rotating counterpart.

Why do we deduce that the primary star exhibits a differential and not a solid rotation? The answer is simple: whereas EK Cep A is both less luminous and smaller than its nonrotating model, a model with solid rotation has lower luminosity and larger radius than a non-rotating model of the same mass, age and chemical composition. Therefore, in the case of solid rotation, while it is possible to fit the luminosity of the model to the observed luminosity (if the difference is not too 
large) by assigning a certain value for angular velocity, this is not the case for the radius. Unlike the luminosity, the radius of a rotating model can be either smaller or larger than that of a non-rotating model of the same kind, depending on the distribution of angular momentum. If however a model has a very slowly rotating envelope but a rapidly rotating interior, then its radius is smaller than that of its non-rotating counterpart, because of its cooler interior.

Our approach to construct a rotating model is very similar to that of Kippenhahn et al. However, we also take into account the rotational kinetic energy in the equation of thermal balance (Eryurt-Ezer et al. 1986) and the evolution of the rotating model is started from the threshold of stability point at which solid-body rotation with $\omega_{0}$ is assumed. As a result of contraction, a radiative interior forms and differential rotation sets in.

To complete our fitting procedure we need to determine $\omega$ as a function of radius at the age of $26 \mathrm{Myr}$. In the outer part, the rotational velocity of EK Cep A can be taken as the synchronous velocity (Tomkin 1983). Then we have only two unknowns, namely $\omega_{0}$ which is linearly related to $\omega$ of the central regions, and the mass fraction of the synchronized outer region $\left(M_{\mathrm{s}}\right)$. They can be derived from the solution of two available equations, analogous to Eqs. (6) and (7), one for luminosity and one for radius, respectively. The relevant numerical derivatives are computed as

$\frac{\partial \log L}{\partial \log \omega_{0}}=-0.25, \quad \frac{\partial \log L}{\partial \log M_{\mathrm{s}}}=0.17$,

and

$$
\frac{\partial \log R}{\partial \log \omega_{0}}=-0.18, \quad \frac{\partial \log R}{\partial \log M_{\mathrm{S}}}=0.06 \text {. }
$$

By this method we find that the central region of the rotating model of EK Cep A with SET E rotates 65 times faster than the surface: while angular velocity of the convective core is $1.3 \times 10^{-3} \mathrm{rad} \mathrm{s}^{-1}$, the outer $48 \%$ of the star's mass rotates with $2.0 \times 10^{-5} \mathrm{rad} \mathrm{s}^{-1}$ (surface equatorial velocity $=22 \mathrm{~km} \mathrm{~s}^{-1}$ ). An interesting point to be noted here is that the effective temperature of the model is higher, in spite of its lower luminosity and smaller radius, than that of its non-rotating counterpart.

If we solve the same equations for the model of EKCep A with SET B, then we find that the central region should rotate 29 times faster than the surface and outer $10 \%$ of its mass is synchronized.

How can we decide which of these solutions is correct? In the next section, we will see that apsidal motion analysis discriminates between these models with very different chemical compositions.

\section{Results and discussions}

\subsection{Apsidal motion of the system}

To obtain the apsidal motion rate, in addition to the published photoelectric eclipse timings in Giménez \& Margrave (1985) and Claret et al., we have adopted the minima given by Bozkurt (2000), Lacy (1998), Lacy et al. (1998), Hegedüs et al. (1996)

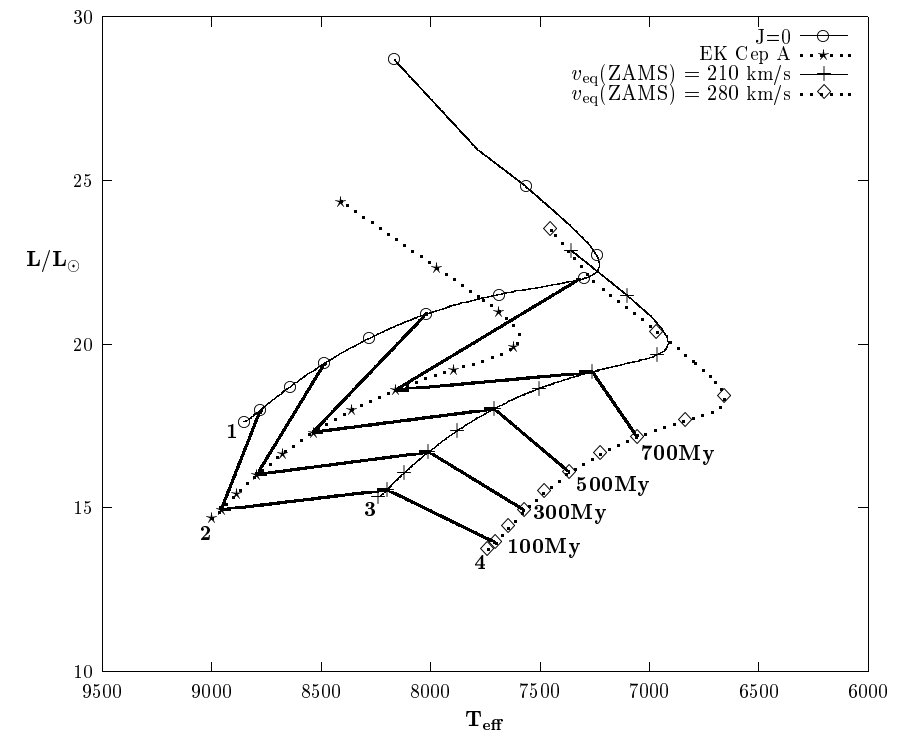

Fig. 4. Main sequence models for a mass of $2.02 M_{\odot}$ with different angular momentum. While Model 1 has no rotation, Model 2 is the differentially rotating model which seems to be the solution for EK Cep A. Equatorial velocity of Model 3 (4) on the ZAMS is $210 \mathrm{~km} \mathrm{~s}^{-1}\left(280 \mathrm{~km} \mathrm{~s}^{-1}\right)$.

(excluding one eclipse due to its large scatter), Ogloza (1995), Lacy et al. (1995) and Lacy \& Fox (1994). Using 38 times of the minima, the observed apsidal advance rate, in which contribution of the classical term is about $55 \%\left(\dot{\omega}_{\text {cl,obs }}=0.95 \times\right.$ $\left.10^{-5} \mathrm{rad} / \mathrm{cyc}\right)$, is found as $1.72 \times 10^{-5} \pm 0.14 \times 10^{-5} \mathrm{rad} / \mathrm{cyc}$ $(\equiv 0.00098 \pm 0.00008 \%$ cyc). This rate is in good agreement with previously found values, but with much less uncertainty.

This observed apsidal motion rate can be used, in principle, to validate both the internal mass distribution and the internal rotation profile of the two components. But in the case of EK Cep, the contribution of the secondary to this rate is so dominant, that not much can be learned about the primary. The agreement is excellent with the metal rich composition SET E, from which we find $\dot{\omega}_{\mathrm{cl} \text {,theo }}=0.95 \times 10^{-5} \mathrm{rad} / \mathrm{cyc}$ at time $26 \mathrm{Myr}$, which is exactly the same as the observed $\dot{\omega}_{\mathrm{cl}, \text { obs }}$. For SET F, this quantity is $\dot{\omega}_{\mathrm{cl} \text {,theo }}=0.92 \times 10^{-5} \mathrm{rad} / \mathrm{cyc}$, slightly less than $\dot{\omega}_{\mathrm{cl}, \text { obs }}$. While models with SET B (the solar metallicity) gives $\dot{\omega}_{\mathrm{cl} \text {,theo }}=0.82 \times 10^{-5} \mathrm{rad} / \mathrm{cyc}$, which is about the lower limit of $\dot{\omega}_{\mathrm{cl}, \mathrm{obs}}$, the metal poor solution (SET A) is never in agreement with the observed apsidal advance rate, and thus we can safely discard it.

\subsection{Consequences of rotation on the age of open clusters and its possible connection with chemically peculiar stars}

In this study, convective overshoot and semi-convection are not taken into account, because the masses of the component stars and the age of the system are small enough (see, e.g., Bressan 1993).

The widening of the top of the MS in the HRD of open clusters is considered as the most important evidence for overshooting. However, it is already known that the same effect can be 
produced by rotation, not only at the top, but also in the lower lying portion of the MS (Talon et al. 1997; Maeder \& Meynet 2000). This fact is again illustrated in Fig. 4 in which the MS evolution of the models with mass of EK Cep A is plotted in the theoretical HRD, with the estimated chemical composition and various angular momentum contents, which are assumed to remain constant on the MS. Thick solid lines represent several isochrones and the first point of each model marks the position of the ZAMS, which depends on the angular momentum content of that model.

In Fig. 4, Model 1 (circles) has no rotation and is always the brightest of all models. Hence, it has the shortest lifetime on the MS (780 Myr). Model 2 (stars) being the best model for EK Cep A has the longest lifetime (970 Myr). It is also the hottest of the models, not only on the ZAMS, but also during the later MS evolution. The equatorial velocity of Models 3 (pluses) and 4 (diamonds) on the ZAMS are $210 \mathrm{~km} \mathrm{~s}^{-1}$ and $280 \mathrm{~km} \mathrm{~s}^{-1}$, respectively, which are close to the observed maximum equatorial velocity for normal A-type stars. Their angular momenta are supposed to be conserved also in the PMS phase. Since rotation slows down the evolution of stars, the MS lifetime of a star depends on its angular momentum. While Model 3 resides on the MS for 870 Myr, Model 4 stays there $80 \mathrm{Myr}$ more. As a result of expansion, the equatorial velocity of both Models 3 and 4 near the end of the MS phase becomes $70 \mathrm{~km} \mathrm{~s}^{-1}$ less than the ZAMS values.

The width of the MS in Fig. 4, determined by these rotationally very different models, corresponds to a temperature difference larger than $1000 \mathrm{~K}$ and is well consistent with that observed in open clusters, even when the binary feature is disregarded. In the unevolved part of the MS (see the ZAMS points and isochrone line for $100 \mathrm{Myr}$ ) the rapidly rotating models are found in the cooler part of the MS as a result of an enlarged radius and the hotter part consists of models with a slowly rotating surface, independent of core rotation. The same is also valid for later times, except for non-rotating Model 1. Due to its rapid evolution, Model 1 evolves faster than the other models toward the cool part of the MS, such that, at $700 \mathrm{Myr}$, when it is about to leave the MS, its effective temperature is almost the same as that of rapidly rotating Model 3 . The example shows that the determination of the age of any open cluster is more difficult and that non-rotating models give merely a lower limit.

It is well known that Ap and Am stars are relatively slowly rotating stars. Although the binarity is supposed to be the principal condition for the formation of Am stars (Böhm-Vitense 1989), we know from the observations that $40 \%$ of them are single stars (North et al. 1998). Am stars, similar to non rotating stars, seem to be more evolved (therefore their models have higher $Z$ than the others) than the normal A type stars of the same kind, maybe due to their very low angular momentum.

Hubrig et al. (2000) confirm that magnetic Ap stars are concentrated toward the center of the MS band, hence they conclude that these stars must have completed at least $30 \%$ of their MS lifetime. However, this concentration is perhaps caused by a structural difference between normal A type and magnetic Ap stars, arising from the steep differential rotation and/or magnetic field in the outer regions of the latter (see Yildiz
2003). If this is the case, then, there should be a connection between peculiar stars and their angular momentum content.

\section{Conclusion}

Binary systems such as EK Cep provide excellent test cases of stellar evolution theory. We constructed a model for EK Cep B which satisfies perfectly the observational constraints at the age of $26 \mathrm{Myr}$. This agreement was obtained with a mixing-length parameter $\alpha=1.30$, which is less than the solar value (1.88), i.e., the mixing length of convective cells in EK Cep B envelope is about $70 \%$ of the mixing length in the solar convective zone. However, what determines the value of this parameter, and how it depends on mass and chemical composition as well as on the evolutionary phase of a star remains an unsolved problem in stellar astrophysics, despite of recent progress (see, e.g., Ludwig et al. 1999, and references therein).

In contrast to the conclusion of Claret et al. on the chemical composition, we have shown that the system abounds in heavy elements $(Z \sim 0.04)$ and our finding is consistent with the observed chemical evolution in the solar neighborhood.

While we can find a solution for EK Cep B within the standard stellar evolution theory, this is not the case for EK Cep A because the observed values for radius and luminosity are less than even the ZAMS model with the composition we have derived through our procedure (SET E). To explain the low radius and luminosity, we suggest for SET E that its central regions rotate 65 times faster than the surface (at $\omega_{\mathrm{c}}=1.3 \times 10^{-3} \mathrm{rad} \mathrm{s}^{-1}$ ) while the outer part, $48 \%$ in mass, is synchronized with the orbital motion $\left(\omega_{\mathrm{s}}=2.0 \times 10^{-5} \mathrm{rad} \mathrm{s}^{-1}\right)$. The values have been derived assuming that the outer part rotates uniformly and the rotational profile of the inner part is determined by the contraction; in reality, some exchange of angular momentum must have occurred, and these figures will be modified accordingly, with a smooth transition between the two regions.

We show also that the differential rotation gives a real widening of the MS in the HRD of open clusters, which corresponds to a temperature difference of about $1000 \mathrm{~K}$. This width is in a very good agreement with the observations and not obtainable using the models with overshooting or models with usual rotational properties.

To validate our model of EK Cep A through the apsidal motion test, it is necessary to improve the precision of eclipse timing. Furthermore, it would be of prime importance to determine the accurate composition of the components. The same is required for all those binary systems (such as GG Lup, PV Cas, IQ Per) with observationally very well known components and relatively small apsidal motion period.

The reason for the diversity observed in early type stars may be that they differ in their angular momentum content and distribution. Possible connections between angular momentum distributions and nature of Ap stars could be verified by similar studies on eclipsing binaries which consist of such stars. In this respect, PV Cas with its very short apsidal motion period is a very appropriate system (Y1ld1z 2003), while further photometric and spectroscopic observations of HD 121276 and AR Aur systems are required for the precise determination of fundamental properties of their components. 
Acknowledgements. I thank Jean-Paul Zahn for his very stimulating discussions and suggestions, Johannes Andersen for providing the observational data and for valuable discussions on eclipsing binaries and Jørgen Christensen-Dalsgaard for his discussions on the early version of the paper. Zeynep Bozkurt is acknowledged for providing her unpublished secondary eclipse timing. I am grateful to my friend Gökhan Sevim for his suggestions which improved the language of the manuscript. This research is supported, in part, by Ege University Research Fund (grant 2000/FEN/055)

\section{References}

Alexander, D. R., \& Ferguson, J. W. 1994, ApJ, 437, 879

Andersen, J. 1991, A\&ARv, 3, 91

Andersen, J., Clausen, J. V., \& Gimenez, A. 1993, A\&A, 277, 439

Bressan, A., Fagotto, F., Bertelli, G., \& Chiosi, C. 1993, A\&AS, 100, 647

Böhm-Vitense, E. 1958, Zs. Ap. 46, 108

Böhm-Vitense, E. 1989, Introduction to Stellar Astrophysics, (Cambridge University Press), Vol. 1

Bozkurt, Z. 2000, private communication

Caughlan, G. R., \& Fowler W. A. 1988, Atomic Data and Nuclear Data Tables, 40, 283

Claret, A., Giménez, A., \& Martín, E. L. 1995, A\&A, 302, 741

Claret, A. 1997, A\&A, 330, 533

Ebbighausen, E. G. 1966, AJ, 71, 642

Eryurt-Ezer, D., Kırbıyık, H., \& Özkara, N. 1986, Ap\&SS, 126, 29

Giménez, A., \& Margrave, T. E. 1985, AJ, 90, 358

Goldreich, P., \& Nicholson, P. D. 1989, ApJ, 342, 1075

Harris, G. M., Roberts, J. E., \& Trulio, J. G. 1960, Phys. Rev., 119, 1832

Hegedüs, T., Bíró, I. B., Borkovits, T., \& Paragi, Z. 1996, IBVS, No. 4340

Hubrig, S., North, P., \& Mathys, G. 2000, ApJ, 539, 352

Iglesias, C. A., Rogers, F. J., \& Wilson, B. G. 1992, ApJ, 397, 717
Kippenhahn, R., Meyer-Hofmeister, E., \& Thomas, H.-C. 1970, A\&A, 5, 155

Lacy, C. H. S., \& Fox G. W. 1994, IBVS, No. 4009

Lacy, C. H. S., İbanoğlu C., Tunca Z., et al. 1995, IBVS, No. 4194

Lacy, C. H. S. 1998, AJ, 115, 809

Lacy, C. H. S., Clem, J. L., Zakirov M., et al. 1998, IBVS, No. 4597

Ludwig, H.-G., Freytag, B., \& Steffen, M. 1999, A\&A, 346, 111

Maeder, A., \& Meynet, G. 2000, ARA\&A, 38, 143

Martín, E. L., \& Rebolo, R. 1993, A\&A, 274, 274

Mihalas, D., Hummer, D. G., Mihalas, B. W., \& Däppen, W. 1990, ApJ, 350, 300

North, P., Ginestet, N., Carquillat, J.-M., Carrier, F., \& Usry, S. 1998 [astro-ph/9805014]

Ogloza, W. 1995, IBVS, No. 4263

Pols, O. R., Tout, C. A., Schroder, K.-P., Eggleton, P. P., \& Manners, J. 1997, MNRAS, 289, 869

Popper, D. M 1987, ApJ, 313, L81

Ribas, I., Giménez, A., Torra, J., Jordi, C., \& Oblak, E. 1998, A\&A, 330, 600

Talon, S., Zahn, J.-P., Maeder, A., Meynet, G. 1997, A\&A, 322, 209

Tomkin, J. 1983, ApJ, 271, 717

Tosi, M. 1995 [astro-ph/9512096]

Yildiz, M. 2000, in Variable Stars as Essential Astrophysical Tools, Proceeding of NATO-ASI, ed. C. İbanoğlu (Dordrecht: Kluwer Academic Pub.), 169

Yıldız, M., \& Kızıloğlu, N. 1997, A\&A, 326, 187

Y1ldı, M. 2003, A\&A, submitted

Zahn, J.-P. 1977, A\&A, 57, 383 